\title{
Pacemaker dysfunction after car accident - a case study.
}

Michał Kowara' ${ }^{1, \mathrm{~A}-\mathrm{D}}$, Agnieszka Kołodzińska ${ }^{2, \mathrm{~A}, \mathrm{C}, \mathrm{E}-\mathrm{F}}$, Marcin Grabowski ${ }^{2, \mathrm{C}, \mathrm{E}-\mathrm{F}}$, Przemysław Stolarz ${ }^{2, \mathrm{~B}-\mathrm{C}}$, Klaudia Wołynkiewicz ${ }^{2, B-C}$, Arkadiusz Brodowski ${ }^{2, B-C}$, Grzegorz Opolski²,E-F

A - Research concept and design, B - Collection and/or assembly of data, C - Data analysis and interpretation,

D - Writing the article, E - Critical revision of the article, F - Final approval of article

1. 1st Department of Cardiology, Medical University of Warsaw, Department of Experimental and Clinical Physiology, Medical University of Warsaw

2. 1st Department of Cardiology, Medical University of Warsaw

Address for correspondence:

Michał Kowara, 1st Department of Cardiology Medical University of Warsaw email: michal.kowara@wum.edu.pl

Agnieszka Kołodzińska, 1st Department of Cardiology, Medical University of Warsaw email: aa.kolodzinska@wp.pl

Marcin Grabowski, 1st Department of Cardiology, Medical University of Warsaw email: grabowski.marcin@me.com

Przemysław Stolarz, 1st Department of Cardiology, Medical University of Warsaw email: p.stolarz@wum.edu.pl

Klaudia Wołynkiewicz, 1st Department of Cardiology, Medical University of Warsaw email: klaudiawolynkiewicz@gmail.com

Arkadiusz Brodowski, 1st Department of Cardiology, Medical University of Warsaw email: cardiology@wum.edu.pl

Grzegorz Opolski, 1st Department of Cardiology, Medical University of Warsaw email: grzegorz.opolski@wum.edu.pl

Received: 25.11 .2017

Revised:

Accepted: 28.11.2017

DOI: $10.24255 / \mathrm{hbj} / 80853$

\section{Key words:}

pacemaker, ineffective pacing, IPG magnet test

\section{Abstract}

A 59-year-old female patient referred to Pacemaker Control Center after car accident, in which pacemaker has been potentially damaged by belt in the car. Although device parameters (threshold, lead impedance and sensing) were stable in time and suggested no damage, an IPG test has revealed no pacing - only sensed sinus rythm was observed. Pacing activity was present only a few minutes after application of „Batery and

\section{Case description}

59-year-old woman was admitted to the Accident and Emergency Department due to a car accident and discharged when major disturbances had been excluded. However, the patient presented to the Pacemaker Control Center three days later because she claimed that during the accident the pacemaker had been squeezed by the safety belt for a few seconds (Figure 1). lead measurement" procedure and became lost shortly after. Therefore the device has been considered as damaged and reimplantation was required. The reimplantation has been undertaken of pacemaker control after potential shock (like in case of car accident) and the role of IPG test in the control.

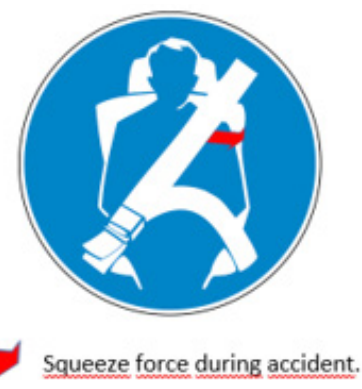


The first pacemaker was implanted in 1993 because of syncope due to second degree atrioventricular block (for technical reasons only a single ventricular lead was implanted and the VVI mode was programmed), then reimplantations were performed in 2005 and 2016 because of the elective replacement indicator (ERI). On 12 September 2016, a new device, Medtronic Sensia SESR01, was implanted. During the pacemaker check just after the accident, pacemaker threshold, lead impedance and sensing test parameters were stable in time (Figure 2).

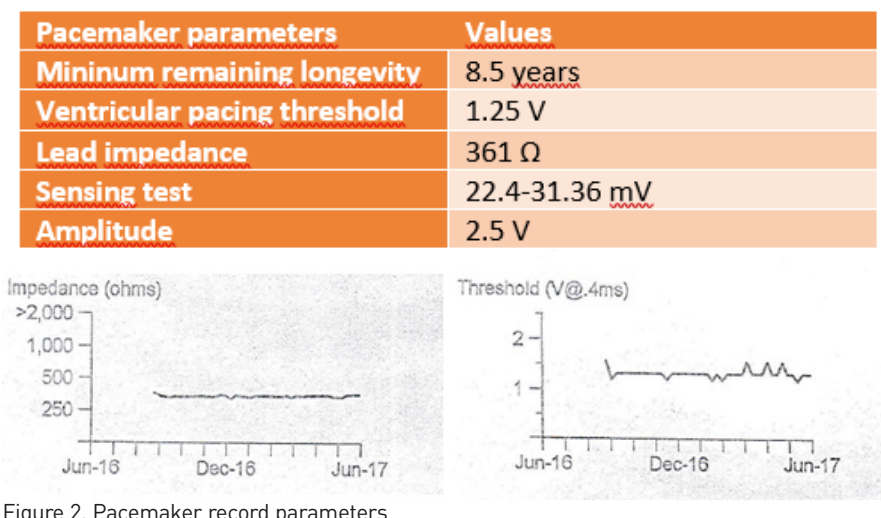

The ventricular long-term histogram (Figure 3) showed that $98.4 \%$ of beats were sensed (only $1.6 \%$ paced), predominantly with a rate below $80 / \mathrm{min}$. It means that the patient was pacemaker-independent.

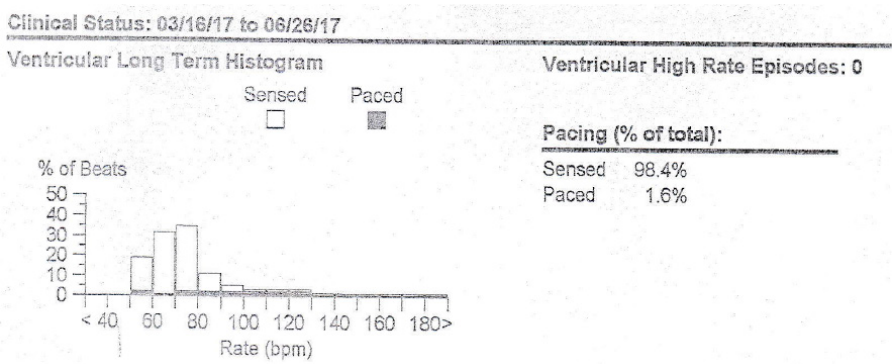

Figure 3. Ventricular long-term histogram records

In addition, the device pocket localized in the left subclavicular region was normal, without any signs of injury or hematoma. However, during the magnetic Implantable pulse generator (IPG) test, pacing was ineffective, and only sensed beats were recorded. After that, the lower tracking rate (LTR) was set to $90 / \mathrm{min}$ in order to check whether the permanent paced rhythm program is accessible. Although the LTR was set to $90 / \mathrm{min}$ and a paced rhythm was supposed to be introduced, no pacing beats were recorded - only sensed beats with a rate $<90 / \mathrm{min}$. Interestingly, after performing the "Battery and Lead measurement" procedure, paced rhythm with a rate of 90/ min was recorded, according to the LTR program. However, after a few minutes pacing episodes became more and more ineffective (Figure 4).

When the patient was turned from a supine to a lateral position, the pacing was lost, and only sensed beats (sinus rhythm, $\mathrm{HR}=70{ }^{\prime}$ ) were recorded. All aforementioned procedures are illustrated in Figure 5.

The opinion from the manufacturer's support center was that the pacemaker was irreversibly damaged during the

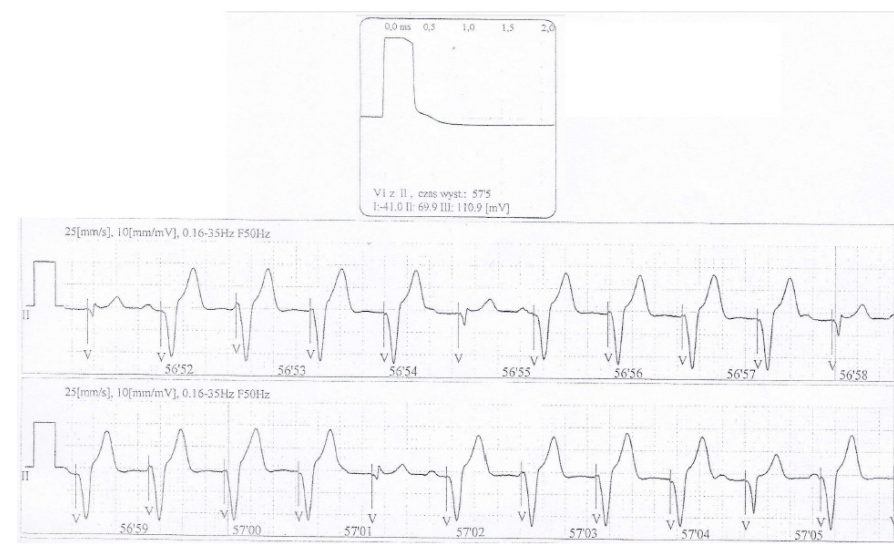

Figure 4. Pacing rhythm HR = 90/min (programmed LTR $=90 / \mathrm{min}$ ) with alternated pacing episodes.

accident and device replacement is warranted. Although the subject was pacemaker independent (Figure 3), the device had been implanted 24 years earlier because of syncope in the course of AV second degree block. Therefore the clinical situation was classified as potentially life-threatening and the patient was transferred to the Accident and Emergency Department and later admitted to the Department of Cardiology. The pacemaker was successfully reimplanted. An X-ray examination and electronic tests during the intervention allowed lead malfunction to be excluded. After the intervention the pacemaker was checked and the patient was discharged the following day.

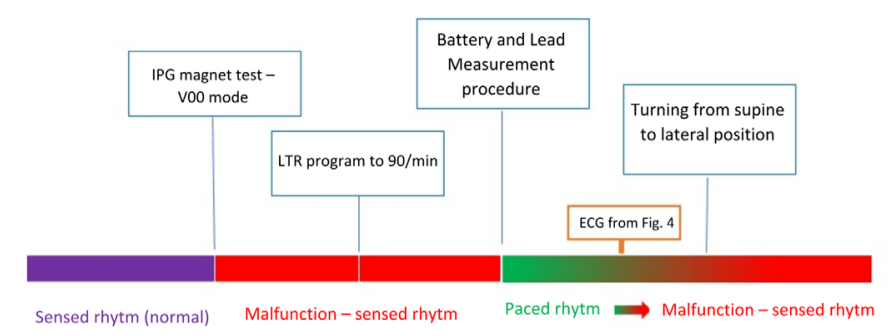

Figure 4. Procedures executed in order to reveal pacemaker dysfunction.

\section{Comment}

Forces generated during a car accident (such as a car collision) can cause damage to a pacemaker. PubMed database research has revealed that it is unknown how many cases of such dysfunction occur during a year. Some national guidelines (for instance Spanish) for drivers indicate that there are no driving restrictions for non-dependent pacemaker patients [1]. However, every suspicion of potential damage (especially after a car accident) should be verified in the Pacemaker Control Center. Our case shows that the IPG magnet test allowed the presence of damage to be revealed. It underlines the importance of this test in the standard pacemaker checkup protocol. Therefore the IPG test should be undertaken routinely during the pacemaker checkup. 


\section{References}

1. García-Cosío Mir F1, Alberca Vela T, Rubio Sanz J, Grande Ruiz A, Viqueira Caamaño JA, Curcio Ruigómez A, Navarro Ruiz V; Sociedad Española de Cardiología. [Guidelines of the Spanish Society of Cardiology for car driving, airplane flying, and underwater activities in subjects with heart disease]. Rev Esp Cardiol. 2001 Apr;54(4):476-90. 